\title{
Spin and Magnetic Field Effects in Chemistry and Related Phenomena
}

\author{
Art van der Est
}

Published online: 7 April 2010

(C) Springer 2010

This special issue of Applied Magnetic Resonance contains a selection of papers reporting work that was presented at the 11th International Symposium on Spin and Magnetic Field Effects in Chemistry and Related Phenomena. ${ }^{1}$ The symposium was held at Brock University in the Niagara Region of Southern Ontario in Canada from the 9-14th of August, 2009. This conference, also known as the Spin Chemistry Meeting (SCM), was first held at Tomakomai, Japan in 1991 and subsequent meetings were held at Konstanz, Germany (1992), Chicago, USA (1993), Novosibirsk, Russia (1995), Jerusalem, Israel (1997), Emmetten, Switzerland (1999) Tokyo, Japan (2001), Chapel Hill, USA (2003), Oxford, UK (2005) and Venice, Italy (2007).

The field of spin chemistry is broadly defined as the study of the influence of electron and nuclear spins on the rates and yields of chemical reactions. Initially, it was dominated by studies of light-induced electron and nuclear polarization generated by radical reactions. However, it has expanded tremendously over the past decade or so and now includes topics as diverse as bird navigation, spintronics, para-hydrogen-induced nuclear polarization and quantum computing. Magnetic resonance methods play a prominent role in these studies and most of the papers in this issue involve electron paramagnetic resonance, nuclear magnetic resonance or other magnetic resonance methods. Readers are also referred to an excellent collection of papers on dynamic nuclear polarization presented in a recent special issue of Applied Magnetic Resonance (2008 volume 34, numbers 3-4).

\footnotetext{
1 The article, E. Daviso, A. Diller, P. Gast, A. Alia, J Lugtenburg, M. G. Müller, J. Matysik, action spectroscopy on dense samples of photosynthetic reaction centers of Rhodobacter sphaeroides WT based on nanosecond laser-flash ${ }^{13} \mathrm{C}$ photo-CIDNP MAS NMR. Appl. Magn. Reson. 38, 105-116 (2010) was also presented at the SCM 2009.
}

A. van der Est $(\bowtie)$

Department of Chemistry, Brock University, 500 Glenridge Ave, St. Catharines,

ON L2S 3A1, Canada

e-mail: avde@brocku.ca 
The SCM 2009 brought together approximately 110 participants from 15 countries and there were many graduate students and postdoctoral fellows who attended. The discussions were very lively and the general consensus among participants was that it was an excellent opportunity to exchange ideas and broaden their perspectives on the field. The diversity of spin chemistry was well represented at the meeting and this is reflected in the articles presented here.

I would like to extend my thanks to all of the authors who have contributed to this issue. We are also grateful to our sponsors (The ACS Petroleum Research Fund, Bruker Canada, PTI Inc, TRC Inc, Wilmad LabGlass, VWR and Brock University). Without their generous support, the conference would not have been possible. 